Bundesgesundheitsbl 2020 - 63:813-820 https://doi.org/10.1007/s00103-020-03171-2 Online publiziert: 10. Juni 2020

(c) Der/die Autor(en) 2020

\section{Dirk Schnabel}

SPZ für chronisch kranke Kinder/Pädiatrische Endokrinologie, Charité - Universitätsmedizin Berlin, Berlin, Deutschland

\section{Biopsychosoziales Betreuungs- konzept für Kinder mit X-chromo- somaler Hypophosphatämie (XLH)}

\section{Beispiel für den multiprofessionellen Ansatz Sozialpädiatrischer Zentren}

\section{Einleitung}

Kinder- und Jugendliche mit einer seltenen, schweren, komplexen chronischen Erkrankungbenötigen in der ambulanten Versorgung neben einer hoch spezialisierten medizinischen Expertise auch die Möglichkeit einer professionellen psychosozialen Unterstützung.

Das Angebot der Versorgung chronisch kranker Kinder und Jugendlicher mit mehrdimensionaler Störung in einem Sozialpädiatrischen Zentrum (SPZ) richtet sich nach dessen lokalen Gegebenheiten. Dabei sind die Fachlichkeit sowie die räumlichen und personellen Erfordernisse zu berücksichtigen. Für SPZ, die die sozialpädiatrische Dimension einer chronischen Erkrankung inhaltlich und personell abdecken können, nicht aber über die Expertise für Grunderkrankungen verfügen, kann zum Beispiel über eine Kooperation mit medizinischen Einrichtungen und Institutionen, die entsprechende Spezialsprechstunden anbieten, diese Versorgung gewährleistet werden. Diese Einrichtungen/Institutionen können Kinderkliniken angegliedert sein oder es handelt sich um universitäre Einrichtungen mit entsprechenden Spezialsprechstunden oder auch niedergelassene spezialisierte Fachärzte für Kinderund Jugendmedizin mit Subspezialisierung bzw. Kinder- und Jugendpsychiatrie.
Sozialpädiatrische Zentren für chronisch kranke Kinder und Jugendliche, angegliedert zumeist an universitäre Kinderkliniken, können das für diese besondere Patientengruppe erforderliche biopsychosoziale Betreuungskonzept sicherstellen [1].

Am Beispiel der im Folgenden genauer beschriebenen Multiorganerkrankung X-chromosomale Hypophosphatämie (XLH) wird später das multiprofessionelle SPZ-Betreuungskonzept dargestellt.

\section{Symptome der XLH}

Die XLH ist mit einer Prävalenz von 1 auf 20.000 bis 25.000 Neugeborene eine seltene Erkrankung [2]. Das führt dazu, dass diese Patienten oftmals eine mehrjährige medizinische Odyssee hinter sich gebracht haben, bevor sie diagnostiziert werden. Die Symptome der Erkrankung sind durchaus unterschiedlich, zumeist steht aber ein verspätetes Laufalter, vergesellschaftet mit einer progredienten Beinachsenfehlstellung (OBein, Genua vara), im Vordergrund. Aber auch Zahnfisteln und Zahnabszesse und ein nachfolgender vorzeitiger Zahnverlust bei einem kariesfreien Gebiss oder ein progredienter, disproportionierter Kleinwuchs können bei einer XLH auftreten $[3,4]$. Da die Säuglinge und Kleinkinder meist noch recht regelmäßig zu den Vorsorgeuntersuchungen beim Kinder- bzw. Hausarzt vorgestellt werden, kommt diesen die Lotsenfunktion im Gesundheitssystem zu.

Klinische Symptome, wie zum Beispiel progrediente Beinachsenfehlstellung, auffälliges breitbasiges Gangbild, Auftreibungen an den Handgelenken, Wachstumsstörung, ggf. auch schon Zahnabszesse oder Zahnfisteln sowie radiologische Auffälligkeiten mit Auffaserung und Becherung an den Wachstumsfugen, weisen auf das Vorliegen einer metabolischen Knochenerkrankung hin. Die laborchemische Konstellation aus zumeist nur leicht erhöhter alkalischer Phosphatase (ALP), aber deutlich erniedrigtem Serumphosphat, bei normalem 25-Hydroxy-Vitamin-D und Parathormon und einem deutlich pathologisch verminderten tubulären Transportmaximum für Phosphat (TmP/GFR) erlaubt dann die Verdachtsdiagnose hypophosphatämische Rachitis. Die Diagnosesicherung erfolgt über die Bestimmung des PHEX-Gens (Phosphate-regulating Gene with Homologies to Endopeptidases on the $X$-chromosome; $[5,6])$.

\section{Pathophysiologie}

Inaktivierende Mutationen im PHEXGen führen zu einer verminderten Expression und gestörten Funktion des PHEX in den Osteozyten, Osteoblasten 


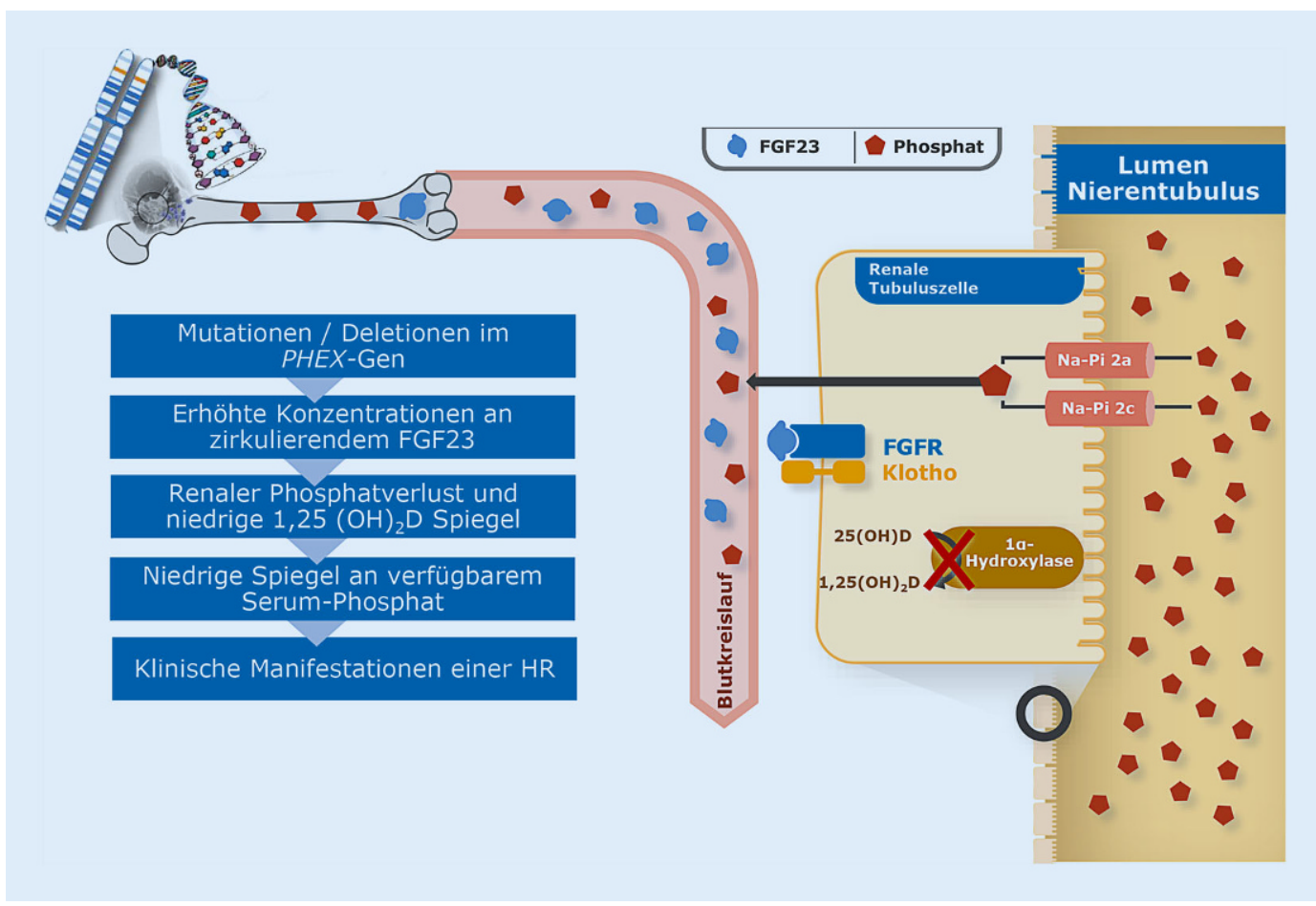

Abb. $1<$ Pathophysiologie der X-chromosomalen Hypophosphatämie (XLH) und Odontoblasten mit der Folge einer Akkumulation von Inhibitoren der Mineralisation, wie z.B. ASARM-Peptiden und Osteopontin (OPN) in bereits kalzifizierter Knochenmatrix, sowie einer vermehrten Synthese und Sekretion des Fibroblastenwachstumsfaktors 23 (FGF23; [7]).

Das FGF23 hat eine zentrale Rolle in der Regulation der Phosphathomöostase. Unter den erhöhten Konzentrationen des zirkulierenden FGF23 wird die Phosphatrückresorption über die Beeinflussung der Funktion und Expression der Natrium-Phosphat-Co-Transporter in der Niere gestört und es kommt zu einem vermehrten renalen Verlust von Phosphat. Das erhöhte FGF23 hemmt zudem die Umwandlung von 25-Hydroxyvitamin $\mathrm{D}$ in das aktive 1,25-Dihydroxyvitamin D, sodass der betroffene Organismus nicht in der Lage ist, über eine verstärkte Calcitriolbildung vermehrt Phosphat enteral zu resorbieren und damit den vermehrten renalen Phosphatverlust zu kompensieren (• Abb. 1). Als Folge des Phosphatmangels kommt es bei der XLH zu einer Mineralisierungsstörung der Knochen und der Zähne betroffener Kinder mit einer Vielzahl klinischer und organischer Auswirkungen auf den Gesamtorganismus, sodass die XLH der
Kategorie der Multiorganerkrankungen zuzuordnen ist.

Die Erkrankung wird zumeist X-chromosomal-dominant vererbt [8].

\section{Medikamentöse Therapie}

Bis zur Zulassung einer zielgerichteten Therapie mit einem FGF23-Antikörper im Jahr 2018 bestand die konventionelle Therapie der XLH aus der Substitution von Phosphat und der Zugabe von aktivem Vitamin D. Die Dosierung von Phosphat beträgt täglich $20-40 \mathrm{mg} / \mathrm{kg}$ Körpergewicht $(\mathrm{KG})$, welches wegen seiner kurzen Halbwertszeit und besseren Verträglichkeit auf mindestens 5 Einzelgaben über den Tag hinweg verteilt werden sollte. Die zusätzliche Einnahme von Calcitriol in einer Dosierung von täglich 20-30 ng/kg Körpergewicht, verteilt auf 1-2 Einzeldosen pro Tag [9], alternativ wegen der besseren Dosierbarkeit besonders bis zum Schulkindalter von Alfacalcidol in der Dosierung 50 ng/kg Körpergewicht pro Tag, dient zur Förderung der enteralen Phosphatresorption über eine Normalisierung des aktiven Vitamins D. $\mathrm{Zu}$ Beginn der Therapie stehen zudem die Linderung von Knochenschmerzen (in den ersten Wochen) und später eine Normalisierung der ALP-Aktivität (nach
6-12 Monaten), eine Verbesserung der Wachstumsgeschwindigkeit (nach einem Jahr) und eine Beinbegradigung (nach 3-4 Jahren) im Vordergrund [5, 10]. Die langfristigen Therapieziele bei Kindern mit XLH sind vor allem das Erreichen eines normalen Knochenumsatzes mit einer vollständigen Mineralisation des Skelettsystems, eine altersgerechte Zahnmineralisation sowie eine Endgröße im Normalbereich.

Die bisherige konventionelle medikamentöse Therapie der XLH ist mit verschiedenen Herausforderungen und $\mathrm{Ne}$ benwirkungen verbunden. Neben einer häufig begrenzten Wirksamkeit ist die Belastung der Patienten durch die mehrmals tägliche Einnahme der oralen Medikation und die damit verbundene niedrige Therapieadhärenz hervorzuheben. Eine Auswertung von Patientendaten des Odenser Universitätskrankenhauses ergab, dass $33 \%$ der pädiatrischen Patienten mit einer hypophosphatämischen Rachitis eine ungenügende Therapieadhärenz aufwiesen (definiert als Einnahme von 3 oder weniger Phosphateinzeldosen pro Tag).

Trotz der Behandlung leiden viele betroffene Kinder aufgrund verschiedener Beschwerden wie Beinachsenfehlstellung, Kleinwuchs, Knochenschmerzen 
unter einer verminderten Lebensqualität $[8,9]$. Nicht selten sind kinderorthopädische Interventionen notwendig [11]. Darüber hinaus birgt die Therapie langfristig das Risiko für verschiedene renale Komplikationen wie Nephrokalzinose, Nephrolithiasis und Hypertonus [12, 13].

Basierte die bisherige Standardtherapie der XLH auf dem Versuch, den renalen Phosphatverlust durch die Einnahme von aktivem Vitamin D und Phosphat soweit zu kompensieren, dass der Knochenumsatz nur wenig gesteigert war, so steht mit der Entwicklung des rekombinanten humanen monoklonalen IgG1-Antikörpers Burosumab nun erstmals ein kausaler Ansatz zur Behandlung der XLH, derzeit noch beschränkt vom 2. Lebensjahr bis zum Schluss der Wachstumsfugen, zur Verfügung.

Burosumab neutralisiert das im Überschuss vorhandene FGF23 und hemmt dadurch dessen Aktivität am FGF23Rezeptor am Nierentubulus [14, 15]: Über die Normalisierung der renalen Phosphatrückresorption kommt es zum Anstieg des Serumphosphats in den unteren Normbereich, im Knochen kommt es konsekutiv über das nunmehr wieder ausgeglichene stöchiometrische Kalzium-Phosphat-Verhältnis zur altersgerechten Mineralisation des Knochens sowie der Zähne. Unterstützt wird der Mineralisierungsprozess noch zusätzlich durch die nunmehr wieder mögliche vermehrte Synthese von Calcidiol zu Calcitriol, welches die Absorption von Phosphat im Dünndarm steigert.

Für die Patienten verbessert sich die Lebensqualität unter der neuen medikamentösen Therapie. Anstatt der bis zu 5-mal täglich erforderlichen Medikamenteneinnahmen wird der spezifische Antikörper nur einmal alle 14 Tage subkutan appliziert. Traten bisher besonders unter der Phosphatsubstitution erhebliche gastrointestinale Nebenwirkungen auf, welche die Adhärenz der regelmäBigen Medikation bestimmten, so sind unter der Antikörpertherapie Akzeptanz und Adhärenz hervorragend. In einer Vergleichsstudie zwischen konventioneller und Burosumab-Therapie [16] fand sich eine signifikante Überlegenheit der neuen zielgerichteten Therapie. Nen-

Bundesgesundheitsbl 2020 $63: 813-820$ https://doi.org/10.1007/s00103-020-03171-2

(c) Der/die Autor(en) 2020

\section{Schnabel}

\section{Biopsychosoziales Betreuungskonzept für Kinder mit X-chromosomaler Hypophosphatämie (XLH). Beispiel für den multiprofessionellen Ansatz Sozialpädiatrischer Zentren}

\section{Zusammenfassung}

Patienten mit einer schweren, seltenen und komplexen Erkrankung benötigen das multiprofessionelle biopsychosoziale Betreuungskonzept eines Sozialpädiatrischen Zentrums für chronisch kranke Kinder und Jugendliche. Am Beispiel der Multiorganerkrankung X-chromosomale Hypophosphatämie (XLH), der häufigsten angeborenen Rachitisform, wird das Betreuungskonzept dargestellt.

Der Erkrankung liegen inaktivierende Mutationen im „Phosphate-regulating gene with Homologies to Endopeptidases on the X-chromosome"(PHEX)-Gen zugrunde, die zu einer vermehrten Synthese und Sekretion des Fibroblastenwachstumsfaktors 23 (FGF23) führen. FGF23 hat eine wichtige Funktion in der Phosphathomöostase. Durch die hohen FGF23-Konzentrationen kommt es über den renalen Phosphatverlust zur schweren Hypophosphatämie mit der Folge erheblicher
Mineralisationsstörungen des Skelettsystems und der Zähne. Stand bis vor Kurzem nur eine konventionelle medikamentöse Therapie aus Phosphat und aktivem Vitamin D zur Verfügung, so ist nun mit dem neutralisierenden FGF23-Antikörper eine gezielte Therapie der Erkrankung möglich. Das multiprofessionelle Betreuungskonzept umfasst zahlreiche ärztliche Spezialisten und ein psychosoziales Team. Ziel der Betreuung ist es, den Patienten mit ihrer schweren chronischen Erkrankung eine altersentsprechende Partizipation ohne größere Teilhabestörung zu ermöglichen. Die Fortsetzung der Betreuung im Erwachsenenalter muss durch eine implementierte Transition sichergestellt werden.

Schlüsselwörter

Multiprofessionelle Betreuung · XLH · Nephrokalzinose $\cdot$ Zahnprobleme $\cdot$ Rachitis

\section{Biopsychosocial care concept for children with X-chromosomal hypophosphatemia (XLH). Example of the multiprofessional approach of social pediatric centers}

\section{Abstract}

Patients with severe, rare and complex diseases require the multiprofessional biopsychosocial care concept of a social pediatric center for chronically ill children and adolescents. The care concept is illustrated using the example of the multiorgan disease X-chromosomal hypophosphatemic rickets $(\mathrm{XLH})$, the most common congenital form of rickets.

The disease is based on inactivating mutations in the Phosphate-regulating gene with Homologies to Endopeptidases on the $\mathrm{X}$-chromosome (PHEX) gene, which leads to an increased synthesis and secretion of fibroblast growth factor 23 (FGF23). FGF23 plays an important role in phosphate homeostasis. High FGF23 concentrations lead to severe hypophosphatemia via renal phosphate loss, resulting in significant mineralization disorders of the skeletal system and teeth. Until recently, only conventional drug therapy consisting of phosphate and active vitamin D was available. Now, the neutralizing FGF23 antibody can be used for a targeted therapy of the disease. The multiprofessional care concept consists of numerous medical specialists and a psychosocial team. The aim of the care concept is to enable patients with their severe chronic disease to participate in everyday life in an age-appropriate manner. The continuation of care in adulthood must be ensured by an implemented transition.

\section{Keywords}

Multiprofessional approach $\cdot \mathrm{XLH}$. Nephrocalcinosis · Dental problems · Rickets 
nenswerte Nebenwirkungen fanden sich unter der neuen Therapie nicht.

\section{Die Multiorganerkrankung XLH}

\section{Organspezifische Probleme - Skelettsystem}

Die Patienten fallen zumeist im 2. Lebensjahr durch ein verspätetes oder auch ausbleibendes Laufen, durch zunehmende Beinachsenfehlstellungen und ein charakteristisches Gangbild, „Watschelgang", auf. Dieser resultiert aus der Kombination einer Fehlstellung am coxalen Femurende, einer Coxa vara sowie der Antekurvation des Oberschenkels. Ursächlich dafür ist die Hypophosphatämie, aus der neben einer schweren Mineralisationsstörung des Knochens auch eine Schwäche der beinbetonten proximalen Muskulatur [17, 18] resultiert.

Bei einer Diagnosestellung in den ersten 3 Lebensjahren besteht bei guter Stoffwechseleinstellung in den darauffolgenden 2-3 Jahren eine gute Chance der Ausgradung der Beinachsen. Gemäß dem Betreuungsprotokoll (• Tab. 1) sollen regelmäßige physiotherapeutische Untersuchungen u. a. mit der Beurteilung der Intermalleolar- und Intercondylarabstände, aber auch des Gelenkstatus durchgeführt werden. Eine regelmäßige standardisierte Fotodokumentation ist wichtig und reduziert die Frequenz der Röntgenganzbeinstandaufnahmen, die aber in bestimmten Abständen indikationsbezogen erfolgen sollen [11].

Die Beinachsenfehlstellung führt zur

- Fehlbelastung der Gelenke und

Ausbildung einer präarthrotischen

Deformität,

- Schmerzen,

- Vermeidung von körperlicher Aktivität mit der Folge:

- Hypo-/Atrophie der Muskulatur und

- Adipositas.

Kommt es trotz guter Stoffwechseleinstellung bei den jüngeren Patienten nicht zu einer signifikanten Ausgradung, so ist die temporäre Epiphyseodese als Option in Betracht zu ziehen.

Sind bei den zumeist jugendlichen $\mathrm{Pa}$ tienten Fehlstellungen in mehreren Ebenen und ggf. auch Torsionsfehler vorhan- den, wird eine Operation nach Ilizarov mit Anlage eines Fixateur externe durchgeführt [19].

Der Zeitpunkt chirurgischer orthopädischer Interventionen sollte in enger Abstimmung zwischen Endokrinologie und Orthopädie erfolgen. Bei Patienten unter der neuen FGF23-Antikörpertherapie ist nach 9-12 Monaten von einem gut mineralisierten Knochen auszugehen, der eine hervorragende Grundlage für eine erfolgreiche Operation darstellt.

Das bis vor einigen Jahren praktizierte Vorgehen, die Patienten erst nach Abschluss des Längenwachstums/Schluss der Epiphysenfugen $\mathrm{zu}$ operieren, ist wegen der bis dahin zumeist erfolgten pathologischen Adaptationen der Gelenke verlassen worden.

Konservative orthopädische Maßnahmen zur Begradigung der Achsenfehlstellung, wie z.B. Orthesen oder Lagerungsschienen, haben sich hingegen in den meisten Fällen als nicht erfolgreich erwiesen. Gelegentlich werden sie aber zur Schmerzlinderung eingesetzt.

Bei älteren Patienten, zumeist ab der 5. Lebensdekade, ist zu beachten, dass es allgemein u. a. durch eine Hyperplasie der Fibrochondrozyten in den Bändern und Sehnen, durch Ablagerung von Kalk oder knöcherne Anbauten im Bereich der Wirbelsäule zur Ausbildung einer Spinalstenose mit neurologischen Symptomen (z.B. Lähmungserscheinungen, Empfindungsstörungen) kommen kann. Speziell kommen als Ursachen der Spinalstenose in Betracht: Hypertrophie der Facettengelenke, Verdickung der vertebralen Laminae, Verkalkung der spinalen Ligamente, $z$. B. posteriores longitudinales Ligamentum [20].

\section{Organspezifische Probleme - Zähne}

Das Auftreten von rezidivierenden Zahnabszessen und Zahnfisteln im kariesfreien Milchzahn- und Permanentgebiss kann ein Hinweis auf eine XLH sein [21]. Patienten mit XLH weisen eine Fehlbildung der Zahnhartsubstanz Dentin auf. Histologisch finden sich im Dentin mit dem sogenannten Interglobulardentin nicht vollständig mineralisierte Anteile.
Zur Pathogenese werden 2 Hypothesen angenommen: Durch die PHEX-Mutation kommt es in den Odontoblasten zu einer Fehlbildung des Dentins bzw. kommt es über die ausgeprägte Hypophosphatämie zu einer Mineralisationsstörung der Zahnhartsubstanz (Dentin, Schmelz; [22]).

Es ist durchaus vorstellbar, dass beide Hypothesen ursächlich verantwortlich für die Zahnprobleme sind. Des Weiteren finden sich in der Zahnpulpa sogenannte Pulpenhörner und vergrößerte Pulpenkaven, die eine Pulpainfektion begünstigen. Die eindringenden Bakterien können über eine Infektion des Zahnnervs zum Absterben desselben führen. Über den Wurzelkanal sind entzündliche Veränderungen bis zu Abszessen in der Wurzelspitze möglich. In den jüngeren Jahren sind zumeist die Schneidezähne, während dann bei den älteren XLH-Patienten zumeist die Backenzähne betroffen sind.

Die Beurteilung des Zahnstatus dieser Patienten setzt eine Kenntnis der Erkrankung voraus. Nicht selten werden Zahnabszesse und Zahnfisteln im kariesfreien Gebiss übersehen mit der Folge des frühen Verlustes permanenter Zähne. Prophylaktisch wird den Eltern eine konsequente und intensive Zahnpflege mit dem Auftragen von Fluorid bei ihren Kindern/Jugendlichen empfohlen [23]. Beim Auftreten von Rissen in der Zahnhartsubstanz sollte eine Fissurenversiegelung erfolgen. Bei den Zähnen ist eine enge Zusammenarbeit zwischen dem Endokrinologen und dem Zahnspezialisten unbedingt erforderlich. Die Intervalle der Zahnuntersuchungen sind in - Tab. 1 aufgeführt.

\section{Organspezifische Probleme - Niere}

Als unerwünschte Therapiefolge wird bei der XLH in 33-80 \% der Patienten, die mit der konventionellen Therapie behandelt werden, eine Ablagerung von Kalziumsalzen im Parenchym der Niere (Nephrokalzinose) beobachtet [12]. Diese hat zumeist eine multifaktorielle Genese:

- Hyperkalziurie als Folge der Therapie mit aktivem Vitamin D [24],

- Hyperphosphaturie als Folge der hohen Phosphatsubstitution mit tubulä- 


\begin{tabular}{|c|c|c|c|c|}
\hline & 0-5 Jahre & 6-10 Jahre & Pubertät & Transition \\
\hline Intervalle der Vorstellungen & Alle 1-3 Monate & Alle 3-6 Monate & Alle 3 Monate & - \\
\hline $\begin{array}{l}\text { Auxologie: Körperhöhe, Gewicht, Kopfumfang, } \\
\text { Körperproportionen, Blutdruck }\end{array}$ & $\checkmark$ & $\checkmark$ & $\checkmark$ & $\checkmark$ \\
\hline Laboruntersuchungen $^{\mathrm{a}}$ & $\boldsymbol{V}$ & $\checkmark$ & Alle 3-6 Monate & $\checkmark$ \\
\hline Radiologische Diagnostik & $\begin{array}{l}\text { Bei Erstdiagnostik, danach } \\
\text { nach Bedarf }\end{array}$ & Nach Bedarf & Nach Bedarf & $\boldsymbol{V}$ \\
\hline Nierensonographie & $1 \times$ jährlich & $1 \times$ jährlich & $1 \times$ jährlich & $\checkmark$ \\
\hline $\begin{array}{l}\text { Physiotherapie: } \\
\text { Prüfung der muskuloskelettalen Funktionen, } \\
\text { 6-Minuten-Gehtest, IMA }, I C A^{\mathrm{c}}\end{array}$ & - & $\checkmark$ & Alle 3-6 Monate & $\boldsymbol{\sim}$ \\
\hline Orthopädie & \multicolumn{3}{|c|}{ Jährlich bei signifikanten Beinachsenfehlstellungen } & $\checkmark$ \\
\hline Kardiologie: Echokardiographie, Langzeit-RR & \multicolumn{3}{|l|}{ Bei RR-Werten >95. Perz. } & $\checkmark$ \\
\hline Zahnarzt & $\begin{array}{l}2 \times \text { jährlich nach Zahndurch- } \\
\text { bruch }\end{array}$ & \multicolumn{2}{|l|}{$2 \times$ jährlich } & $\boldsymbol{V}$ \\
\hline HNO/Gehör & - & \multicolumn{2}{|c|}{$\begin{array}{l}\text { Ab dem 8. Lebensjahr: Prüfung des Hörvermögens } \\
\text { Wiederholung bei V.a. Hörstörung }\end{array}$} & $\checkmark$ \\
\hline Lebensqualität/Psychologie & $\begin{array}{l}\text { Eltern (zu jedem Zeitpunkt) } \\
\text { Angebot Psychologie }\end{array}$ & \multicolumn{2}{|c|}{ Ab dem 8. Lebensjahr jährlich } & $\checkmark$ \\
\hline Soziale Arbeit & Nach Bedarf & \multicolumn{2}{|l|}{ Nach Bedarf } & $\checkmark$ \\
\hline \multicolumn{5}{|c|}{$\begin{array}{l}\text { Anmerkung: Die Notwendigkeit der Untersuchungen richtet sich neben den allgemeinen Empfehlungen nach der Klinik des einzelnen Patienten und } \\
\text { können deshalb variieren } \\
\text { In der Titrationsphase des Medikaments Crysvita sollten die in der Produktinformation empfohlenen Blutentnahmeintervalle beachtet werden. Zusätzlich } \\
\text { ist die Bestimmung von 1,25(OH) } 2 \text { D nur unter Crysvita sinnvoll } \\
\text { aAP, S-Phosphat, S-Kalzium, Kreatinin, 25-OHD, Parathormon, TmP/GFR, U-Kalzium, U-Phosphat, U-Kreatinin } \\
{ }^{b} \text { ICA Intercondylarabstand } \\
\text { 'IMA Intermalleolarabstand }\end{array}$} \\
\hline
\end{tabular}

ren Kalzium-/Phosphatausfällungen [25],

- Hyperoxalurie als Folge der Phosphatsubstitution [26],

- sekundärer Hyperparathyreoidismus als Folge der Phosphatsubstitution [27],

- FGF23 stimuliert über die enteralen TRPV5-Kanäle im Übermaß die zelluläre Aufnahme von Kalzium [28, 29].

Berichteten Stickler und Morgenstern [30] noch über 3 behandelte Patienten, die terminal niereninsuffizient wurden, so sind in den letzten 2 Jahrzehnten keine derartigen Verläufe beschrieben [31]. Auch ausgeprägte Nephrokalzinoseformen enden zumeist nicht in einer Nierenfunktionseinschränkung. Allerdings gibt es Berichte über das erhöhte Hypertonusrisiko. Dieses könnte allerdings auch noch zusätzlich durch die nicht unerhebliche Adipositas mitgetriggert werden, welche die Patienten zumeist ab dem Pubertätsalter entwickeln. Die nunmehr initiierten Patientenregister unter Einschluss der erwachsenen $\mathrm{Pa}$ tienten werden hoffentlich Aussage zur Langzeitnierenfunktion der Patienten geben.

Zur möglichen Verhinderung der Ausbildung einer Nephrokalzinose werden die konsequente Aufteilung der Phosphatdosen über den Tag, das Einhalten eines $<0,6 \mathrm{mmol} / \mathrm{mmol}$ Kreatinin, ein normales Parathormon und ein $\mathrm{Ab}$ setzen der Medikation bei Immobilisation empfohlen. Die Kontrollintervalle zur Nierenfunktion sind in - Tab. 1 dargestellt. Möglicherweise wird unter der zielgerichteten FGF23-Antikörpertherapie das Nephrokalzinoserisiko kaum noch eine Rolle spielen, wenngleich eine abschließende Bewertung der unter dieser Therapieform beobachteten $\mathrm{zu}$ meist erhöhten 1,25-DihydroxyvitaminD-Konzentrationen noch nicht vorliegt.

\section{Organspezifische Probleme - Schädel}

Der prämature Verschluss der Schädelnähte, Kraniosynostose, führt zu ei- nem ungleichmäßigen Schädelwachstum und einer auffälligen Kopfform, z. B. Trigonocephalus bei Frontalnahtsynostose. Es können einzelne, aber auch mehrere Nähte gleichzeitig von der vorzeitigen Verknöcherung betroffen sein. Bei einem Teil der Patienten kann es über einen erhöhten intrakraniellen Druck zur Verlagerung der Kleinhirntonsillen in das Foramen magnum (Chiari-I-Malformation) kommen. Neben kognitiven Störungen, Verhaltensauffälligkeiten, Sehstörungen oder chronischen Kopfschmerzen kann eine Kraniosynostose zu ästhetischen Symptomen wie auffälligen Kopfformen oder Gesichtsdeformationen führen.

Rothenbuhler et al. [32] fanden in ihrer XLH-Kohorte von 44 Patienten bei $59 \%$ eine komplette oder partielle Fusion der Sagitalnaht und bei $25 \%$ einen Kleinhirntonsillentiefstand. Eine neurochirurgische Intervention erfolgte bei $9 \%$ der 44 Patienten. Als Ursache der vorzeitigen Fusion der Schädelsuturen wird eine Cross-Bindung von FGF23 mit FGFR2 und FGFR3 disku- 


\section{Infobox 1 Physiotherapie- konzept bei X-chromosomaler Hypophosphatämie (XLH) \\ Eingangsuntersuchung: \\ - Bestimmung der Achsabweichungen (Malleolar-/Interkondylarabstand) \\ - Gelenkstatus \\ - Muskelkraftmessung (Sprungplatte, Handdynamometer) \\ - Gangdokumentation \\ - 6-Minuten-Gehtest \\ Anleitung zum häuslichen Training: \\ - Stärkung der Glutealmuskulatur \\ - Stärkung der Rückenmuskulatur \\ - Stärkung der Bauchmuskulatur \\ - Übungen auf dem Balancebrett (Stärkung Bein-/Rumpfmuskulatur, Verbesserung der Koordination) \\ - Übungen zur Verbesserung der Gelenksta- bilität}

Unterstützende Maßnahmen:

- Physiotherapie (fakultativ)

- Vibrationstraining („Galileo")

Kontrolluntersuchungen alle 3-6 Monate - Siehe Eingangsuntersuchung

tiert. Da Kraniosynostose und Chiari-IMalformation mit der Schwere der XLH assoziiert sind [33], eine Korrelation zwischen einer Kraniosynostose und aufgetretenen Zahnabszessen besteht, ist davon auszugehen, dass die Entwicklung der kranialen Komplikationen vom Serumphosphat abhängen.

Die Erfassung des Kopfumfanges sowie die Beurteilung der Schädelform sollten regelmäßig durchgeführt werden (•Tab. 1). Kopfschmerzen, Sehstörungen, Schlafstörungen, Übelkeit/ Erbrechen, Parästhesien oder Kleinhirnsymptome sollten unverzüglich eine radiologische Diagnostik (MRT, ggf. 3-D-CCT) nach sich ziehen.

\section{Physiotherapeutisches Konzept}

Die Einbeziehung der Physiotherapeuten erfolgt bereits zum Zeitpunkt der Diagnosestellung (s. • Infobox 1). Dabei sollten im Rahmen einer ausführlichen Statuserhebung das Ausmaß der Beinachsenfehlstellung, die erhöhte bzw. eingeschränkte Gelenkbeweglichkeit, Muskelkraft, Koordination und körperliche Belastbarkeit, z.B. mittels des
6-Minuten-Gehtestes, erfasst werden. Bei den XLH-Patienten finden sich aufgrund der ausgeprägten Hypophosphatämie eine Mineralisationsstörung des Skelettsystems mit Achsenfehlstellungen der unteren Extremitäten sowie eine zum Teil erhebliche Muskelschwäche mit deutlicher Einschränkung der körperlichen Belastbarkeit $[17,18]$. Spezielle Ganganalysen der Kölner KinderKnochen-Arbeitsgruppe um Schönau/ Semler/Starke zeigen, dass die Hüfte der Patienten relativ stabil bleibt, das watschelnde Gangbild vor allem aus dem Rumpfpendeln resultiert. Darüber hinaus finden sich aber noch weitere Muskelgruppen der unteren Extremitäten mit einer reduzierten Muskelkraft. Die Patienten sollten je nach Beteiligung der im Vordergrund stehenden Muskelgruppen und instabilen Gelenke und ggf. zusätzlich vorhandener Koordinationsstörung ausführliche (schriftliche) Anleitungen zum häuslichen Training (• Abb. 2) erhalten. Dabei sind mögliche Hemmnisse der Aktivitätssteigerung, wie Disproportionierung und Adipositas, bei der individuellen Trainingsplanerstellung zu berücksichtigen. Die Notwendigkeit zur zusätzlichen Verordnung einer professionellen Physiotherapie und/oder eines Vibrationstrainings ist individuell $\mathrm{zu}$ entscheiden.

\section{Soziale Arbeit}

Ein nicht unerheblicher Anteil der Patienten ist körperlich in seinen Alltagsaktivitäten eingeschränkt. Mitarbeiter der sozialen Arbeit können die Eltern über die Möglichkeit des Antrages aufSchwerbehinderung (Für und Wider) beraten und ggf. Hilfestellung bei Antragstellung leisten (insbesondere bei Analphabeten oder Sprachbarrieren), aber auch eine Abschätzung der Erfolgsaussichten einer solchen Antragsstellung geben. Die Beratung der Eltern über den Integrationsstatus in Kita bzw. Schule, die Unterstützung in Behördenangelegenheiten (Schuldnerberatung, Jugendamt, Sozialamt, Ausländerbehörde etc.), aber auch das Anfertigen von Stellungnahmen für die öffentlichen Gesundheitsdienste sind mögliche Aufgaben der Sozialarbeiter. Über die Möglichkeit der Zuordnung der
Patienten zum Personenkreis $₫ 53$ Abs. 1 SGB XII durch das SPZ haben die Eltern das Recht auf Eingliederungshilfen. Eine wichtige Unterstützungsmöglichkeit stellt z. B. die Einzelfallhilfe dar, die aber leider nicht in allen Bundesländern verfügbar ist. Aufgaben und Ziele der Einzelfallhilfe sollten sein:

- Aktivierung zu Alltagsbewegung,

- Förderung der sportlichen Aktivitäten,

- Förderung der Selbstwahrnehmung und Selbstständigkeit,

- Begleiten bei Alltags- und Entwicklungsprozessen,

- Förderung sozialer Kontakte und Interaktionen,

- Unterstützung in Schule und Freizeitgestaltung.

\section{Psychologie}

Die Berufsgruppe der Psychologen ist integraler Bestandteil des multiprofessionellen Betreuungskonzepts chronisch kranker Kinder im SPZ. Es ist sinnvoll, sie möglichst schon in das Erstgespräch bei Diagnoseeröffnung mit den Eltern und den Patienten einzubeziehen. Die Familie soll von Anfang an begleitet und unterstützt werden, um den Verarbeitungsprozess der Krankheit günstig $\mathrm{zu}$ beeinflussen und unerwünschten sekundären Krankheitsfolgen frühzeitig entgegenwirken $\mathrm{zu}$ können. Treten in bestimmten Lebensphasen Probleme mit der Adhärenz u.a. der z.T. lebensnotwendigen Einnahme/Applikation von Medikamenten auf, so ist die Einbeziehung psychologischer Unterstützung unbedingt sinnvoll. Einzelgespräche, therapeutische Vereinbarungen oder Verhaltensübungen könnten dann zur Problemlösung beitragen. Des Weiteren gehören zum Betreuungsangebot der Psychologen in Abhängigkeit vom Lebensalter die sozial-emotionale und kognitive Diagnostik im Vorschulalter, eine Schulleistungsdiagnostik sowie die Diagnostik und Schullaufbahnberatung. 


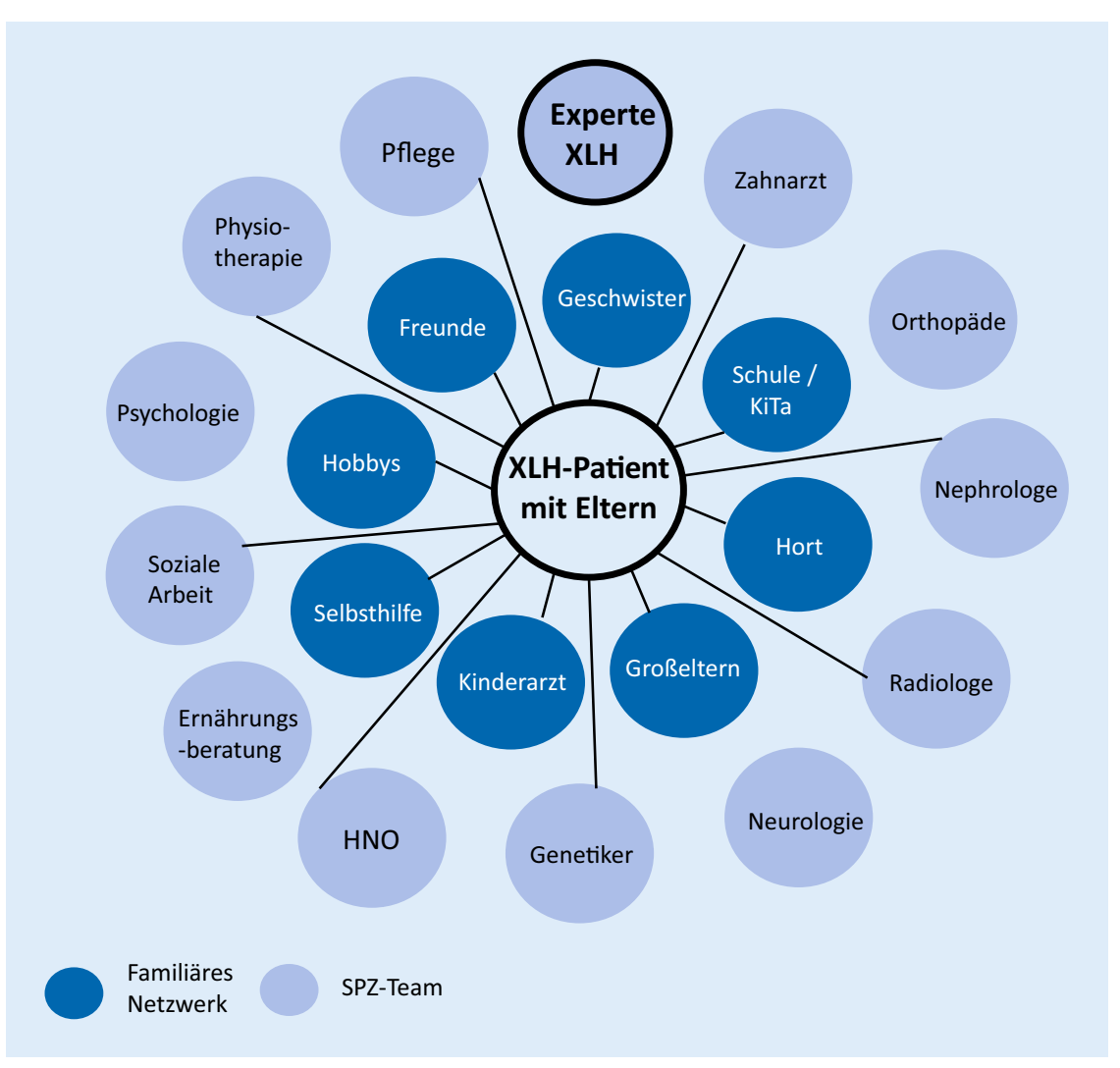

Abb. $2 \Delta$ Der kindliche/jugendliche Patient mit X-chromosomaler Hypophosphatämie $(X L H)$ mit seinem familiären Netzwerk sowie den Professionen des multidisziplinären Teams im Sozialpädiatrischen Zentrum (SPZ). Der Arzt und Experte für die Erkrankung XLH leitet und koordiniert das SPZ-Team

\section{Ziele der multiprofessionellen Behandlung der Patienten im SPZ}

Durch das multiprofessionelle Behandlungskonzept für Patienten mit komplexen chronischen Erkrankungen im SPZ (- Abb. 2) für chronisch kranke Kinder und Jugendliche sollte erreicht werden [34]:

- die Sicherstellung eines weitgehend selbstbestimmten Lebens des Kindes und der Familie, die Stärkung seiner Eigenaktivität sowie eine altersentsprechende Partizipation,

- die Verbesserung der Qualität der medizinischen und therapeutischen Behandlung und der psychosozialen Betreuung,

- die Verringerung von Komplikationen bei chronischen Erkrankungen mit mehrdimensionalen Störungen,

- in der Langzeitbetreuung eine Reduktion kostspieliger stationärer Krankenhausaufenthalte,
- die Integration in die Gesellschaft und in ein nachhaltiges, altersentsprechendes Umfeld einschließlich der Berufsfindung.

\section{Transition in die Erwachsenen- betreuung}

Für Patienten mit einer XLH gibt es bisher noch kein standardisiertes Transitionsverfahren, wie es für Diabetes mellitus Typ 1 oder komplexe hypothalamischhypophysäre Erkrankungen im Berliner Transitionsprogramm bereits vorliegt [35]. Dies liegt u. a. daran, dass es bis zur Publikation einer europäischen Konsensusgruppe [36] keine nationalen bzw. internationalen Konsensusempfehlungen zur konzeptionellen Weiterbetreuung der Patienten im Erwachsenenalter gab. Klinische Studien an erwachsenen XLH-Patienten aus der letzten Zeit zeigen aber, dass die Patienten aufgrund der z.T. ausgeprägten gesundheitlichen Folgen ihrer Erkrankung (u.a. Pseudofrakturen, Knochen-/Gelenkschmerzen, deutlich verminderte körperliche Belastbarkeit, Enthesopathien, Osteoarthritis; [37]) unbedingt eine Fortsetzung der medikamentösen Therapie als auch ein multiprofessionelles Betreuungskonzept benötigen. Im Zuge der erfolgreichen Implementierung eines sinnvollerweise an die Fachgesellschaften angegliederten XLH-Patientenregisters für das Kindesund Jugendalter (Deutsche Gesellschaft für Kinderendokrinologie und -diabetologie, DGKED/Gesellschaft für Pädiatrische Nephrologie, GPN) sollte nun auch ein standardisiertes Transitionsverfahren etabliert werden.

\section{Fazit}

Der multiprofessionelle Betreuungsansatz eines SPZ für chronisch kranke Kinder und Jugendliche, exemplarisch dargestellt an der XLH, berücksichtigt neben der (bio-)medizinischen auch die psychosozialen Facetten der schweren Multiorganerkrankung auf universitärem Wissens- und Forschungsstand.

Mit der neuen medikamentösen Therapieoption - Burosumab - sowohl im Kindes- und Jugendalter sowie voraussichtlich auch ab Ende 2020 im Erwachsenenalter [38] wird sich zeigen müssen, ob die bisherigen klinischen Probleme der Patienten, wie z.B. Knochenmineralisationsstörung, vorzeitiger Zahnverlust, Kraniosynostose, disproportionierter Kleinwuchs, zu reduzieren oder gar $\mathrm{zu}$ vermeiden sind.

Das SPZ-Team wird die Patienten sicherlich auch zukünftig begleiten und nicht in seinen Aktivitäten nachlassen, z. B. durch Informationsveranstaltungen für pädagogische Fachkräfte in Kitas und Schulen, das Wissen über chronische Erkrankungen zu erhöhen, und versuchen, Ängste im Umgang mit den ihnen anvertrauten chronisch kranken Kindern und Jugendlichen zu nehmen. Die Verknüpfung von fachlicher Expertise einer zumeist tertiären medizinischen Versorgungseinrichtung mit den Möglichkeiten der Sozialpädiatrie bietet den XLH-Patienten eine realistische Möglichkeit auf eine altersentsprechende Partizipation ohne größere Teilhabestörung. 


\section{Korrespondenzadresse}

\section{Dr. med. Dirk Schnabel}

SPZ für chronisch kranke Kinder/Pädiatrische Endokrinologie, Charité - Universitätsmedizin Berlin

Augustenburger Platz 1 (Mittelallee 7a),

13353 Berlin, Deutschland

dirk.schnabel@charite.de

Funding. Open Access funding provided by Projekt DEAL.

\section{Einhaltung ethischer Richtlinien}

Interessenkonflikt. D. Schnabel ist als Referent und Berater für die Firma Kyowa Kirin $\mathrm{GmbH}$ tätig.

Für diesen Beitrag wurden von den Autoren keine Studien an Menschen oder Tieren durchgeführt. Für die aufgeführten Studien gelten die jeweils dort angegebenen ethischen Richtlinien.

Open Access. Dieser Artikel wird unter der Creative Commons Namensnennung 4.0 International Lizenz veröffentlicht, welche die Nutzung, Vervielfältigung Bearbeitung, Verbreitung und Wiedergabe in jeglichem Medium und Format erlaubt, sofern Sie den/die ursprünglichen Autor(en) und die Quelle ordnungsgemäß nennen, einen Link zur Creative Commons Lizenz beifügen und angeben, ob Änderungen vorgenommen wurden.

Die in diesem Artikel enthaltenen Bilder und sonstiges Drittmaterial unterliegen ebenfalls der genannten Creative Commons Lizenz, sofern sich aus der Abbildungslegende nichts anderes ergibt. Sofern das betreffende Material nicht unter der genannten Creative Commons Lizenz steht und die betreffende Handlung nicht nach gesetzlichen Vorschriften erlaubt ist, ist für die oben aufgeführten Weiterverwendungen des Materials die Einwilligung des jeweiligen Rechteinhabers einzuholen.

Weitere Details zur Lizenz entnehmen Sie bitte der Lizenzinformation auf http://creativecommons.org/ licenses/by/4.0/deed.de.

\section{Literatur}

1. Schnabel D (2020) Multiprofessionelle Betreuung von chronisch kranken Kindern im Sozialpädiatrischen Zentrum der Charitè Berlin. In: Mall V, Voigt $F$, Jung NH (Hrsg) Sozialpädiatrie - State of the Art und Zukunftsperspektiven. Max Schmidt-Römhild GmbH \& KG, Lübeck, S 115-125

2. Beck-Nielsen SS, Brock-Jacobsen B, Gram J, Brixen K, Jensen TK (2009) Incidence and prevalence of nutritional and hereditary rickets in southern Denmark. Eur J Endocrinol 160:491-497

3. Schnabel D (2018) Knochenmineralisationsstörungen im Kindes- und Jugendalter. Kinder Jugendmed 2:1-6

4. Hiort O, Schnabel D (2019) Endokrine Störungen des Mineralhaushaltes bei Kindern und Jugendlichen. In: Hiort O, Danne T, Wabitsch M (Hrsg) Pädiatrische Endokrinologie und Diabetologie, 2. Aufl.Springer, Berlin, S475-498
5. Linglart A, Biosse-Duplan A, Briot K et al (2014) Therapeutic management of hypophosphatemic rickets from infancy to adulthood. Endocr Connect 3:R13-R30

6. Carpenter TO, Imel EA, Holm IA, de Beur JSM, Insogna KL (2011) A clinician's guide to X-linked hypophosphatemia. JBone Miner Res 26:1381-1388

7. Beck-Nielsen SS, Mughal Z, Haffner D et al (2019) FGF23 and its role in X-linked hypophosphatemiarelated morbidity. Orphanet J Rare Dis 14:58

8. Schnabel D (2018) X-chromosomale Hypophosphatämie. Thieme Drug Rep 12(13):1-12

9. S1-Leitlinie: Hereditäre hypophosphatämische Rachitiden, AWMF-Register-Nr. 174/008, Stand: März 2016

10. Rothenbuhler A, Schnabel D, Högler W, Linglart A (2019) Diagnosis, treatment-monitoring and follow-up of children and adolescents with X-linked hypophosphatemia (XLH). Metabolism. https://doi.org/10.1016/j.metabol.2019.03.009 (Epubahead of print)

11. Correll J (2004) Orthopädische Probleme beim Phosphatdiabetes. In: Mohnike K, Klingebiel KH (Hrsg) Familiäre hypophosphatämische Rachitis. ABM-Wissenschaftsverlag, Berlin, S57-69

12. Beetz R (2004) Organspezifische Problematik Niere. In: Mohnike K, Klingebiel KH (Hrsg) Familiäre hypophosphatämische Rachitis. ABMWissenschaftsverlag, Berlin, S83-89

13. Nakamura $Y$, Takagi $M$, Takeda $R$, Miyai $K$ Hasegawa Y (2017) Hypertension is a characteristic complication of X-linked hypophosphatemia. Endocr J 64:283-289

14. Caballero D, Li Y, Ponsetto J, Zhu C, Bergwitz C (2017) Impaired urinary excretion in Npt2a-/-mice. Am JPhysiol Renal Physiol 312:F77-F83

15. Carpenter TO, Whyte MP, Imel EA et al (2018) Burosumab therapy in children with X-linked hypophosphatemia. NEngl J Med 378:1987-1998

16. Imel EA, Glorieux FH, Whyte MP et al (2019) Burosumab versus conventional therapy in children with X-linked hypophosphataemia: a randomized, active-controlled, open-label, phase 3 trial. Lancet393(10189):2416-2427

17. Veilleux LN, Cheung M, Amor BM, Rauch F (2012) Abnormalities in muscle density and muscle function in hypophosphatemic rickets. J Clin Endocrinol Metab 97:E1492-E1498

18. Veilleux LN, Cheung M, Glorieux FH, Rauch F (2013) Abnormalities in muscle density and muscle function in hypophosphatemic rickets. J Clin Endocrinol Metab 98:E990-E995

19. Kocaoglu M, Bilen FE, Sen C, Eralp L, Balci HI (2011) Combined technique for the correction of lowerlimb deformities resulting from metabolic bone disease. JBone Joint Surg Br 93:52-56

20. Karaplis AC, Bai X, Falet JP, Macica CM (2012) Mineralizing enthesopathy is a common feature of renal phosphate-wasting disorders attributed to FGF23 and is exacerbated by standard therapy in hype mice. Endocrinology 153:5906-5917

21. Hanisch M (2019) Mundgesundheit und Lebensqualität bei Phosphatdiabetes. In: Klein M (Hrsg) Phosphatdiabetes (XLH) und Lebensqualität. Vom Leben mit einer seltenen und rätselvollen Erkrankung, 2. Aufl. Münster, S55-59

22. Osahl Vital S, Gaucher C, Bardet C et al (2012) Tooth dentin defects reflect genetic disorders affecting bone mineralization. Bone 50:989-997

23. Hanisch M, Bohner L, Sabandal MMI, Kleinheinz Jung $S$ (2019) Oral symptoms and oral healthrelated quality of life of individuals with $X$-linked hypophosphatemia. Head Face Med 15(1):8
24. Mitchell DM, Juppner $\mathrm{H}$, Burnett-Bowie SM (2017) FGF23 is not associated with age-related changes in phosphate, but enhances renal calcium reabsorption in girls. J Clin Endocrinol Metab 102:1151-1160

25. Alon U, Donaldson DL, Hellerstein S, Warady BA, Harris DJ (1992) Metabolic and histologic investigation of the nature of nephrocalcinosis in children with hypophosphatemic rickets and in the Hyp mouse. J Pediatr 20:899-905

26. Patzer L, van't Hoff W, Shah V et al (1999) Urinary supersaturation of calcium oxalate and phosphate in patients with X-linked hypophosphatemic rickets and in healthy schoolchildren. J Pediatr 135:611-617

27. Reusz GS, Brodehl J, Krohn HP, Ehrich JH (1990) Hypophosphataemic rickets. Lancet 335:178

28. Andrukhova O, Smorodchenko A, Egerbacher M et al (2014) FGF23 promotes renal calcium reabsorption through the TRPV 5 channel. EMBO J 33:229-246

29. Andrukhova O, Slavic S, Smorodchenko A et al (2014) FGF23 regulates renal sodium handling and blood pressure. EMBO Mol Med 6:744-759

30. Stickler GB, Morgenstern BZ (1991) Hypophosphatemic rickets: final height and clinical symptoms in adults. Lancet 14:902-905

31. Eddy MC, Mc Alister WH, Whyte MP (1997) X-linked hypophosphatemia: normal renal function despite medullary nephrocalcinosis 25 years after transient vitamin D2-induced renal azotemia. Bone 21:515-520

32. Rothenbuhler A, Fadel N, Debza Y et al (2019) High incidence of cranial synostosis and chiari malformation in children with X-linked hypophosphatemic rickets. JBMR 34(3):490-496

33. Di Rocco F, Rothenbuhler A, Cormier Daire V et al (2019) Craniosynostosis and metabolic bone disorder. A review. Neurochirugie 65:258-263

34. Schnabel D, Ernst M, Marton MA et al (2012) Behandlung von chronisch kranken Kindern und Jugendlichen im Sozialpädiatrischen Zentrum. In: Fricke C, Hollmann H, Kretzschmar C, Schmid RG (Hrsg) Qualität in der Sozialpädiatrie, Bd. 3. BAGSPZ, RS-Verlag, Altötting, S240-262

35. Müther S, Findorff J (2016) Berliner Transitionsprogramm. Sektorenübergreifendes Strukturprogramm für die Transition Jugendlicher mit chronischer Erkrankung. Springer-Verlag,

36. Haffner D, Emma F, Eastwood D et al (2019) Clinical practice recommendations for the diagnosis and management of X-linked hypophosphatemia. Nat Rev Nephrol 15(7):435-455

37. Che H, Roux C, Etcheto A et al (2016) Impaired quality of life in adults with $X$-linked hypophos phatemia and skeletal symptoms. Eur J Endocrinol $174: 325$

38. Insogna KL, Briot K, Imel EA et al (2018) A Randomized, Double-Blind, Placebo-Controlled, Phase 3 Trial Evaluating the Efficacy of Burosumab, an Anti-FGF23 Antibody, in Adults With X-Linked Hypophosphatemia: Week 24 Primary Analysis. JBMR 33:1-11 